\title{
ARTICLE
}

Cite this: DOI: 10.1039/x0xx00000x

\section{The many roles of mellitic acid during barium sulfate crystallization}

Received 00th January 2012 Accepted 00th January 2012

DOI: $10.1039 / \times 0 \times x 00000 x$

www.rsc.org/

\author{
Matthew P. Boon ${ }^{\mathrm{a}}$, Sandra Freeman ${ }^{\mathrm{a}}$, Mark I. Ogden ${ }^{\mathrm{a}}$, Allan Oliveira ${ }^{\mathrm{a}}$, William R. \\ Richmond $^{\mathrm{a}}$, Brian W. Skelton ${ }^{\mathrm{b}}$ and Franca Jones ${ }^{\mathrm{a} *}$
}

The various roles of mellitic acid during barium sulfate crystallization from nucleation to

The various roles of mellitic acid during barium
mesocrystal formation is explored and elucidated.

\section{Introduction}

Classical crystallization has provided a basis for understanding the process of how random hydrated ions in solution come together and form ordered crystalline solids. Knowing crystallization theory can, in principle, help us understand how the minerals form on our earth, how Nature forms various biominerals in living organisms and how we may make particles to suit our technological needs. Unfortunately, classical crystallization theory cannot explain many crystallization phenomena ${ }^{1,2}$. In biomineralization, amorphous precipitates are often formed, rather than crystalline nuclei ${ }^{3,4}$. Quite recently, the stabilisation of nanoparticles that aggregate and then form mesocrystals has shown another non-classical crystallization mechanism ${ }^{5}$. In mesocrystal formation growth beyond the nano-scale does not occur; rather the micron-sized particles are formed by the self-assembly of the stabilised nanoparticles into larger structures ${ }^{6,7}$.

There has been much work conducted in the area of mesocrystals and their formation in the presence of polymeric species $^{8-11}$. We have, in the past, shown that small molecules can stabilise barium sulfate and that this is most likely due to self-assembly of the organic molecule $\mathrm{e}^{12-14}$ resulting in the steric stabilisation required. Formation of meso-crystals is also possible by charge stabilisation of nanoparticles ${ }^{15,16}$ rather than steric stabilisation since the general mechanism relies on any form of stabilization of the initial nanoparticle before formation of the mesocrystal and/or fusion to an iso-oriented crystal.

In this work, barium sulfate is used as our crystallizing solid phase. Baryte (barium sulfate solid) has several properties which make it useful as a model system; the first is that the ionic species are known and do not alter speciation significantly even with moderate $\mathrm{pH}$ changes, the other is that only one polymorph of barium sulfate is formed (baryte) even when conditions are significantly altered.

Previous work has shown mellitic acid to be an inhibitor of barium sulfate crystallization, which led to the formation of mesocrystals (see Figure 1) of barium sulfate ${ }^{17}$. Thus, an important aspect of this study was to determine the how mellitic acid is involved in the formation of the meso-crystal. Additionally, infrared studies on this system showed that mellitic acid was intimately associated with the initial nucleation of barium sulfate particles from solution ${ }^{18}$. Thus, mellitic acid was also influencing the nucleation behaviour of barium sulfate. For this reason, the system was investigated to determine what impact the mellitic acid had on all aspects of crystallization and particle formation and to rationalise those impacts based on chemistry and complexation behaviour. The results emphasise that impurities have far reaching impacts that are not always fixed to one particular stage of crystal formation.<smiles>O=C(O)c1c(C(=O)O)c(C(=O)O)c(C(=O)O)c(C(=O)O)c1C(=O)O</smiles>

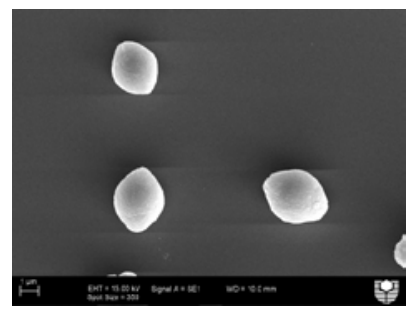

Figure 1. Schematic of mellitic acid (left) and SEM image of baryte solids formed when $0.03 \mathrm{mM}$ mellitic acid is present 


\section{Experimental}

\section{Materials}

The materials used in this study were AR grade, from Ajax Chemicals, Sigma Aldrich or BDH and were dissolved to the required concentrations using filtered $(0.2 \mu \mathrm{m})$, ultrapure $(>18$ $\mathrm{M} \Omega \mathrm{cm}$ resistivity) water.

\section{Methods}

Morphology and electron microscopy. Batch crystallization was undertaken to observe the impact on morphology, the method used was identical to that previously reported in ref 17. Samples from timed experiments were used for transmission electron microscopy investigations as reported in references 17 and 18. Conditions and are detailed in the appendix.

Turbidity. The effect of mellitic acid on the nucleation behaviour of barium sulfate during crystallization was determined by its impact on turbidity. The induction time (time taken for the turbidity to rise above zero) was determined at different mellitic acid concentrations. Induction time changes reflect nucleation rate changes, which in turn can give information about the surface free energy of the nuclei (in classical nucleating systems). The turbidity was measured using a UV-vis instrument (GBC, UV-Vis 916 instrument) operating at $900 \mathrm{~nm}$ to limit absorption of the light by the solution and dissolved species. The crystallization reaction, equilibrated at temperature involved $200 \mathrm{~mL}$ ultrapure water, $0.5 \mathrm{~mL}$ barium chloride solution $(0.1 \mathrm{M})$ and $0.5 \mathrm{~mL}$ sodium sulfate solution $(0.1 \mathrm{M})$ stirred at $150 \mathrm{rpm}$ with a four-pronged glass impellor. If mellitic acid solution ( $1 \mathrm{~g} / \mathrm{L}$ stock solution) was added, the amount of ultrapure water was adjusted to maintain a constant final volume. This solution was pumped through a flowthrough quartz cuvette and the absorbance reading through the cell constantly monitored. The flow rate through the cell was $67 \mathrm{~mL} / \mathrm{min}$ and this was achieved using a Masterflex ${ }^{\circledR}$ peristaltic pump and Tygon ${ }^{\circledR}$ tubing.

AFM. The AFM procedure is the same as that described in reference ${ }^{19}$. A mineralogical sample of barite was freshly cleaved prior to each experiment and the supersaturation index was SI $=25$ The supersaturation calculated in this manuscript uses the PHREEQC program $^{20}$ to calculate the ion activity product (IAP), and then this is used to determine the supersaturation index such that:

$$
\mathrm{SI}=\left(\mathrm{IAP} / \mathrm{K}_{\mathrm{sp}}\right)
$$

A syringe pump (driven at $0.2 \mathrm{~mL} / \mathrm{min}$ ) was used to deliver the solutions to a fluid cell while the AFM software collected images continuously, thereby monitoring crystallization growth in-situ. Growth under conditions sans mellitic acid were first obtained so that all subsequent data could be normalised.

Confocal Raman spectra and maps were obtained on an Alpha 300SAR confocal Raman microscope (WITech, Germany) equipped with a 100x objective (numerical aperture 0.9 ).
Spectra were obtained with a frequency doubled NdYAG laser having a final excitation wavelength of $532 \mathrm{~nm}$ and using an integration time of $50 \mathrm{~ms}$.

Zeta Potential and Size. The zeta potential and particle counts (by dynamic light scattering, DLS) was measured using a Malvern NanoZeta ZS in a disposable zeta cell. Particles were measured in the solution they were formed. This consisted of preparing $20 \mathrm{~mL}$ solutions containing $50 \mu \mathrm{L}$ of the barium chloride $(0.1 \mathrm{M})$ with the required mellitic acid (with adjusted water volume if necessary to maintain $20 \mathrm{~mL}$ total volume). Sodium sulfate $(50 \mu \mathrm{L}, 0.1 \mathrm{M})$ solution was addded to commence crystallization and the vial was then left for three day. After this time, the vial was sonicated gently in a bath sonicator to disperse the particles and an aliquot taken for measurement. For the timed experiments the reactor and conditions used in the turbidity experiments was replicated. At pre-determined intervals an aliquot was taken and the zeta potential or size measured. Although, size measurements were also taken during the timed experiments the data obtained was used to determine the particle counts.

Degree of complexation between mellitic acid and barium ions. The extent to which mellitic acid complexed barium ions in solution at the same $\mathrm{pH}$ as in the conductivity, morphology, AFM and zeta potential experiments was determined in two ways. Firstly, a Ba ion selective electrode (ISE) was used to see whether the addition of mellitic acid to a known barium ion concentration resulted in a lowering of the barium ion activity. Secondly, the barium chloride conductivity was compared to the barium ion conductivity with mellitic acid to see whether there was any change in the mobility of the solution measured.

Crystallography. The solid formed by complexation of barium ions with mellitate ions was obtained by adjusting the mellitic acid $\mathrm{pH}$ to $>9$ using sodium hydroxide. Barium chloride $(0.1$ M) solution was subsequently added at $3 \mathrm{~mol}$ equivalents. Initially, a white cloudy precipitate forms (presumably barium hydroxide) which overnight transforms to clear long needles appropriate for single crystal X-ray structure determination.

Crystallographic data for the structure were collected at 150(2) $\mathrm{K}$ on an Oxford Diffraction Gemini diffractometer fitted with Mo $\mathrm{K} \alpha$ radiation. Following multi-scan absorption corrections and solution by direct methods, the structure was refined against $F^{2}$ with full-matrix least-squares using the program SHELXL-97 ${ }^{21}$. Several peaks in later maps were modelled as solvent water molecules with site occupancy factors constrained to 0.5 or 1.0 after trial refinement. Water molecule hydrogen atoms were not included in the model. All remaining hydrogen atoms were added at calculated positions and refined by use of riding models with isotropic displacement parameters based on those of the parent atoms. Anisotropic displacement parameters were employed throughout for the non-hydrogen atoms. Full details of the structure determination 
have been deposited with the Cambridge Crystallographic Data Centre.

Crystal Data: $\mathrm{C}_{12} \mathrm{H}_{28} \mathrm{Ba}_{3} \mathrm{O}_{26} ; M=1000.36$, monoclinic, $I 2 / \mathrm{a} ; a=$ 13.4109(2), $b=16.2568(4), c=27.9028(6) \AA, V=6008.7(2)$ $\AA^{3}, Z=8 ; D_{c}=2.212 \mathrm{~g} \mathrm{~cm}^{-3}, \mu=3.988 \mathrm{~mm}^{-1}$, crystal size $=$ $0.30 \times 0.035 \times 0.03 \mathrm{~mm}^{3} .59752$ reflections collected, 15427 unique $\left(R_{\text {int }}=0.0816\right)$, max. $/ \mathrm{min}$. transmission $=1.00 / 0.95$. Data $/$ restraints $/$ parameters $=9865 / 0 / 375 ; \mathrm{GoF}=1.039 . R 1$ $=0.0611(I>2 \sigma(I)), w R 2=0.1681$ (all data). Largest diff. peak and hole $=4.206$ and -1.427 e. $\AA^{-3}$. CCDC no. 1032712 .

Thermogravimetry. TGA analysis was conducted on a TA Instruments SDT 2960 simultaneous DSC-TGA from ambient to $900{ }^{\circ} \mathrm{C}$ at $5{ }^{\circ} \mathrm{C}$ per minute in air at a flow rate of $40 \mathrm{ml} / \mathrm{min}$ using nitrogen gas. A known weight of sample (15-50 mg) was heated in a platinum pan for each measurement. The temperature of the instrument was calibrated against the melting points of indium, zinc, tin, silver and gold. The balance was calibrated over the temperature range with standard alumina weights as provided by the vendor.

\section{Results}

\section{Complex formation and analysis by single crystal XRD}

The ability of organic molecules to complex ions in solution is important to the process of crystallization. This is because complexation will lead to changes in the activity of ions and so must be taken into consideration when determining supersaturation. Additionally, by understanding the solution interactions of the ion with the organic molecule it is hoped to better understand the surface interactions.

It was found that mellitic acid complexed insignificant amounts of barium at $\mathrm{pH} 6$ as determined by either the use of the barium ISE or conductivity (conductivity data shown in supplementary information, S1) up to concentrations of $10 \mathrm{ppm}$ or $0.03 \mathrm{mM}$ mellitic acid.

Crystals of barium mellitate were obtained and high resolution single crystal XRD data taken for structure determination. Barium mellitate was found to readily crystallize from mellitic acid solutions of $\mathrm{pH}>9$ at concentrations as low as $0.03 \mathrm{mM}$. When sulfate solution was added, no barium sulfate precipitated despite the supersaturation for barium sulfate being $\sim 16$ even when complete complexation to mellitic acid was assumed. The barium mellitate particles were long and needlelike as can be seen in Figures 2a, and c. The ends did not appear to have sharp well defined faces (Figure 2b) and had glassy type breakages. When the slurry containing barium mellitate particles and sulfate in solution was heated to $60{ }^{\circ} \mathrm{C}$ overnight, barium sulfate particles were seen to precipitate (rhombic particle in Figure 2d).

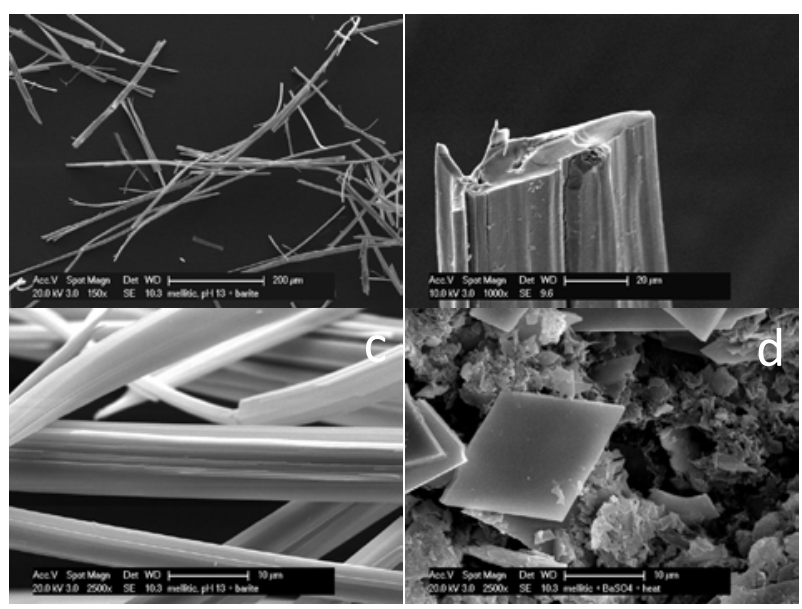

Figure 2. SEM images of a), b) and c) barium mellitate particles and d) barium sulfate particles formed after heating barium mellitate in the presence of sulfate to $60^{\circ} \mathrm{C}$ (see text for further details)

The barium mellitate crystals were of sufficient quality for a single crystal x-ray structure determination, the results of which were consistent with a formulation of $\left[\mathrm{Ba}_{3}(\right.$ mellitate $\left.)\left(\mathrm{H}_{2} \mathrm{O}\right)_{10}\right] .4 \mathrm{H}_{2} \mathrm{O}$. There are three unique barium atoms in the structure, all of which are nine-coordinate with the coordination sphere consisting of carboxylate $\mathrm{O}$ atoms and water molecules, three $\mathrm{H}_{2} \mathrm{O}$ molecules around $\mathrm{Ba} 1$ and $\mathrm{Ba} 3$ and four around $\mathrm{Ba} 2 . \mathrm{Ba} 1$ and $\mathrm{Ba} 3$ form two seven membered chelate rings, bridging adjacent carboxylate groups of the same ligand, as well as a four membered chelate ring to both $\mathrm{O}$ atoms of one carboxylate group. $\mathrm{Ba} 2$ is similar, except that only one seven-membered chelate ring is found, the other being replaced by a unidentate mellitate ligand and the additional water $\mathrm{O}$ atom (Figure S2). As a result, the mellitate anion is bridged across all carboxylate groups aside from group 1 to 6 , with groups 1, 3 and 5 also bound as O,O' bidentate. (Figure S3).

The structure consists of a 3-D polymer with large channels running through the lattice in the $a$ direction (Figure 3, occupying water molecules not shown). The $\mathrm{Ph}$ ring lies almost in the $b c$ plane, the dihedral angle being 8.3 degrees. The $\mathrm{Ba}$ atoms bridge between ligands created by the crystallographic $a$ glide forming stacks along the $a$-axis, (centroid-centroid = $6.7 \AA$ ) This is seen in the cell plot down $a$, supplementary information S4). There are also bonds from the Ba atoms to the $\mathrm{O}$ atoms of other ligands generated by the crystallographic 21 screw along the $b$ axis and by the inversion centres, which create the $3 \mathrm{D}$ coordination polymer. 


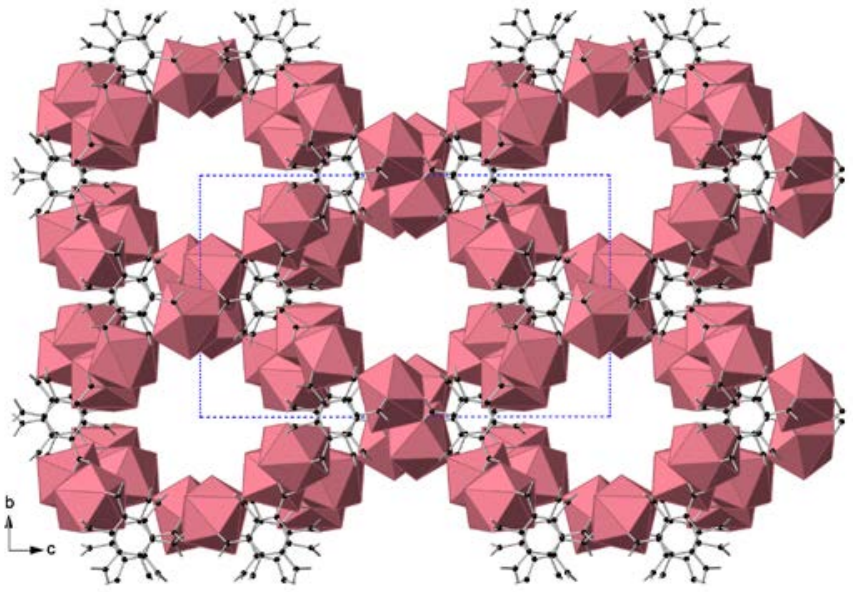

Figure 3. Crystal packing

in

the

$\left[\mathrm{Ba}_{3}\right.$ (mellitate) $\left.\left(\mathrm{H}_{2} \mathrm{O}\right)_{10}\right] .4 \mathrm{H}_{2} \mathrm{O}$ crystal structure. $\mathrm{BaO}_{9}$ centres are represented as polyhedral, and water molecules occupying the channels have been omitted for clarity.

\section{TGA}

The results from the thermogravimetric analysis of the solids are shown in Figure 4. Clearly, little mass is lost from pure barium sulfate at temperatures $\leq 800^{\circ} \mathrm{C}$. The barium mellitate complex loses water up until $100-150^{\circ} \mathrm{C}$ and decomposes above $650{ }^{\circ} \mathrm{C}$. The water loss from barium mellitate is calculated to be $\sim 25.8 \mathrm{wt} \%$. This is only marginally higher than $25.2 \mathrm{wt} \%$ expected from the single crystal data and may suggest some residual surface adsorbed water. The loss of water also suggests that the water found in the barium mellitate structure is weakly bound being driven off at temperatures less than $200^{\circ} \mathrm{C}$.

The barium sulfate particles formed in the presence of mellitic acid at $0.03 \mathrm{mM}$ do not show a plateau in the mass loss above $100{ }^{\circ} \mathrm{C}$. Given that pure barium sulfate does not have a mass loss in this region and that water is being lost for barium mellitate, this would suggest a range of water environments (of differing energy) in this system. In fact, one would additionally conclude that, generally, more energy is required to drive out this water from the barium sulfate structure when formed in the presence of the mellitic acid. It is found that approximately $7.1 \mathrm{wt} \%$ of mass is lost for the baryte particles formed in the presence of mellitic acid. If we assume, just as found in the crystal structure of the complex, that for every molecule of mellitic acid that is present, 14 molecules of water are also present this would equate to 0.03 moles mellitate per mole $\mathrm{BaSO}_{4}$.

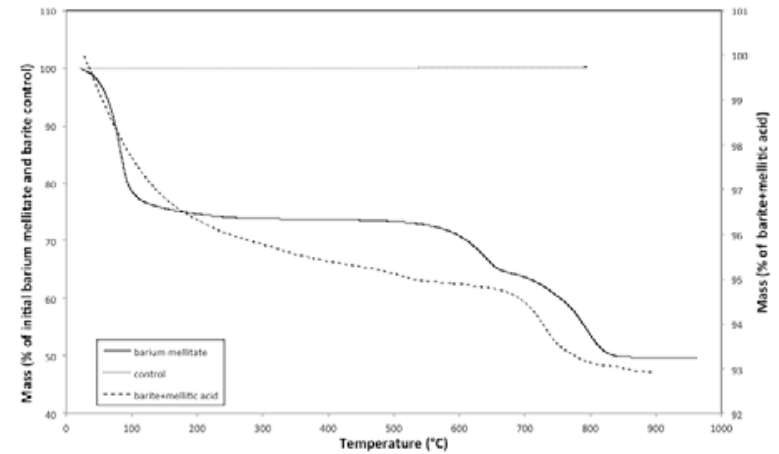

Figure 4. TGA curves for pure barium sulfate, barium mellitate and barium sulfate formed in the presence of mellitic acid.

This is despite the fact that the solution molar ratio of mellitic acid to barium ions was roughly $1.2: 1$. Thus, only a small proportion of the mellitic acids adsorbs or incorporates into the solid structure. In order to determine whether the mellitic acid is mainly adsorbed on the surface or is also incorporated into the particles, the maximum amount of mellitic acid adsorbed was calculated (by assuming the mellitate is not lying flat). For a $50 \mathrm{~nm}$ particle, adsorption on the surface would account for only half the mellitate found in the sample. Thus, it appears that the mellitate ion can both adsorb on the surface of the particles and/or incorporate into the barium sulfate solids.

\section{Zeta Potential}

The zeta potential results for the barium sulfate particles formed in the absence of mellitic acid show a surface potential of less than $-5 \mathrm{mV}$, confirming that these particles essentially carry little to no surface charge (Figure 5). Figure 5 shows the zeta potential measured on barium sulfate particles after three days suspended in the solution in which they were prepared. From this Figure it is observed that the zeta potential undergoes a minimum value of $\sim-30 \mathrm{mV}$ before increasing dramatically to $\sim-10 \mathrm{mV}$ at higher mellitic acid concentrations. Thus, at the minimum zeta potential the barium sulfate particles are on that borderline region between stability and coagulation (it is a common rule of thumb that slurries with surface charges larger, in absolute terms, than $\pm 30 \mathrm{mV}$ are stable). Thus, between 5-10 ppm mellitic acid, the nanoparticles could be charge stabilised. This raises an interesting point. If the particles are within the surface charge range where coagulation is expected outside of this concentration range, why is it that mesocrystals are still able to form? What is the nanoparticle stabilisation mechanism that allows them to self-assemble outside of this concentration range? 


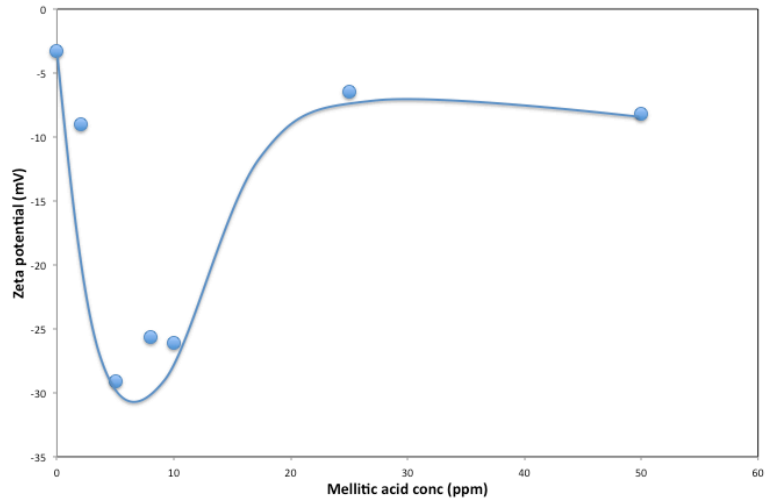

Figure 5. Zeta potential measured on barium sulphate particles after 3 days in the solution they were formed (line drawn to aid reader only)

Perhaps, the answer lies with how the zeta potential evolves over time. At 5 ppm $(0.015 \mathrm{mM})$ mellitic acid, it is clearly seen that the zeta potential of baryte particles decreases quickly over the first 30-60 minutes and then plateaus on longer time scales (Figure 6). The behaviour is similar for the control even if the magnitudes are different. However, when 20 ppm $(0.06 \mathrm{mM})$ mellitic acid was present, initially a slightly lower minimum was found $(\sim-25 \mathrm{mV})$ but this increased over time to $\sim-14 \mathrm{mV}$. Thus, at higher mellitic acid concentrations the surface charge on the barium sulfate particles is only slightly less negative while the steady state value after three days can be significantly less negative.

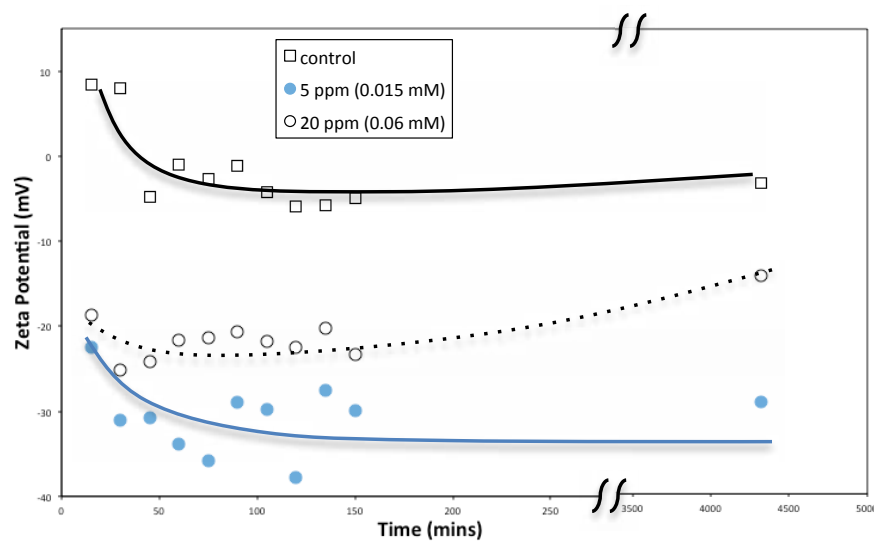

Figure 6. Zeta potential measured on barium sulphate particles versus time in the solution they were formed for 0,5 and $20 \mathrm{ppm}$ mellitic acid present (lines drawn to aid reader only)

This may point to the mesocrystal formation occurring soon after formation of the nanoparticles when the zeta potential is at its most negative.

\section{AFM}

AFM of the growing barium sulfate (001) face in the presence of mellitic acid showed an interesting phenomenon whereby the surface became significantly roughened compared to the control (Figure $7 \mathrm{a}$ and $7 \mathrm{e}, \mathrm{d}$ and the line profile shown in the supplementary information, S4). This made measurement of growth rates and nucleation rates for the 2D islands almost impossible.
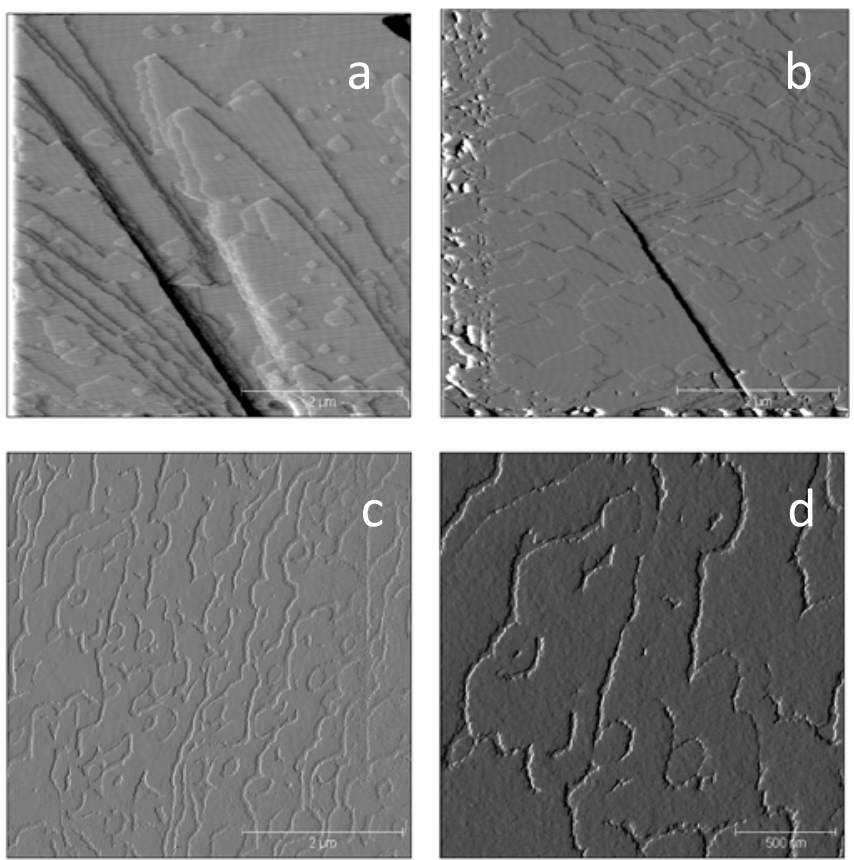

Figure 7. AFM deflection images of the barium sulfate (001) face growing with a) no mellitic acid present, b) $0.1 \mathrm{ppm} \mathrm{c)} 0.2 \mathrm{ppm}$ and d) $0.2 \mathrm{ppm}$ mellitic acid present at a higher magnification. Scale bars are $2 \mu \mathrm{m}$ except for $\mathrm{d}$ ) where it is $500 \mathrm{~nm}$.

In order to determine the distribution of mellitic acid on the surface of the growing particle, confocal Raman mapping was conducted. The amount of mellitic acid on the surface was not sufficient to observe its Raman spectrum over and above that of the barium sulfate Raman spectrum (Figure 8d), however, a broad fluorescence band was observed that was not seen in the control. Mapping of the intensity of the fluorescence proved more sensitive than the Raman spectra and is shown in Figure $8 \mathrm{c}$. The fluorescence was observed on the entire surface but some areas were more fluorescent than others as seen in Figure $8 \mathrm{c}$. Assuming that the intensity of the fluorescence is related to the quantity of adsorbed mellitic acid, it is clear that the mellitic acid is non-uniformly distributed on the surface of the barium sulfate. This distribution of the mellitic acid is broadly consistent with the observations from AFM. 


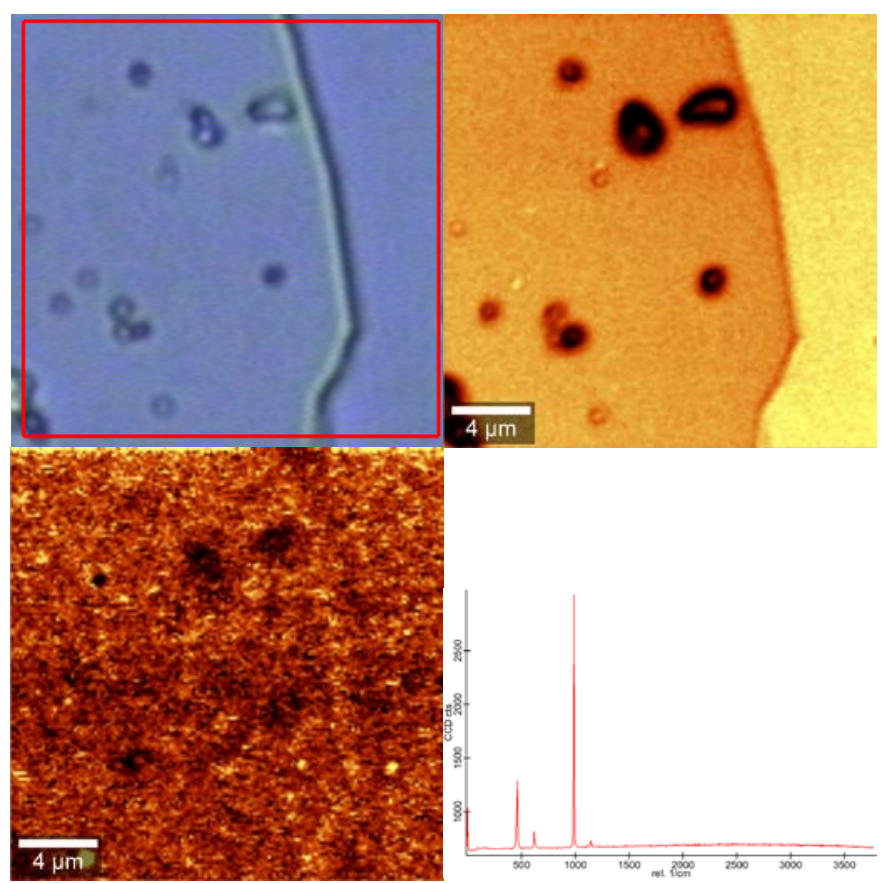

Figure 8. Raman confocal mapping experiment. a) optical image of area mapped outlined by red square, b) map associated with the 990$1000 \mathrm{~cm}^{-1}$ sulfate peak, c) map associated with the fluorescence intensity and d) typical Raman spectrum observed for the area

\section{Discussion}

The mellitic acid is known to be an intergral part of the initially forming nuclei of baryte particles as determined from infrared data previously obtained ${ }^{18}$. Moreover, these particles are initially amorphous and transform over time to crystalline solids ${ }^{18}$ confirming a non-classical pathway for formation of the nuclei. Standard turbidimetric data suggested that the nucleation rate was significantly reduced in the presence of mellitic acid (see supplementary information, S5), however, more sensitive DLS data did not show decreased particle counts in the presence of mellitic acid nor was the onset of the high particle counts delayed when mellitic acid was present (see supplementary information, S6). This can be rationalised by the fact that the standard turbidimetric method fails to detect particles less than $400 \mathrm{~nm}^{22}$, thus 3D nucleation may not be inhibited at all. In addition, the roughness observed in the AFM images when mellitic acid is present suggests that $2 \mathrm{D}$ nucleation is promoted. Thus, while complexation of barium with mellitate is not expected to be significant, the presence of mellitic acid appears to promote the $2 \mathrm{D}$ nucleation rate of barium sulfate.

From the complex formed between barium and mellitate ions it is clear that water is a significant part of the structure and that significant amounts of water are also found in the baryte solids formed. Thus, the formation of amorphous barium sulfate nuclei can be reconciled with the substantial amount of water present meaning the crystallization process would involve removal of this water. This would also correlate with the internal porosity and ageing effects seen for the particles (where some fusion of the mesocrystal form is observed, see Figure 9a) as well as the TGA analysis. From the infrared data we have seen that the organic de-protonates on adsorption ${ }^{18}$ (mellitic acid FTIR peaks are consistent with the fully deprotonated mellitate ion, $\mathrm{L}^{6-}$ ). This behaviour (de-protonation on adsorption) has been previously observed for other carboxylate systems ${ }^{23}$. In addition, the promotion of nucleation by mellitic acid may be due to the association of the barium ions to the mellitate ions after adsorption, especially since dehydration of the barium ions is known to be a rate-limiting step for baryte crystallization ${ }^{24,25}$. It is also seen that while the 'particles' of baryte are $\sim 50 \mathrm{~nm}$ in diameter they appear to be made of smaller particles still, of $\sim 10 \mathrm{~nm}$ in diameter (see Figure 9b).

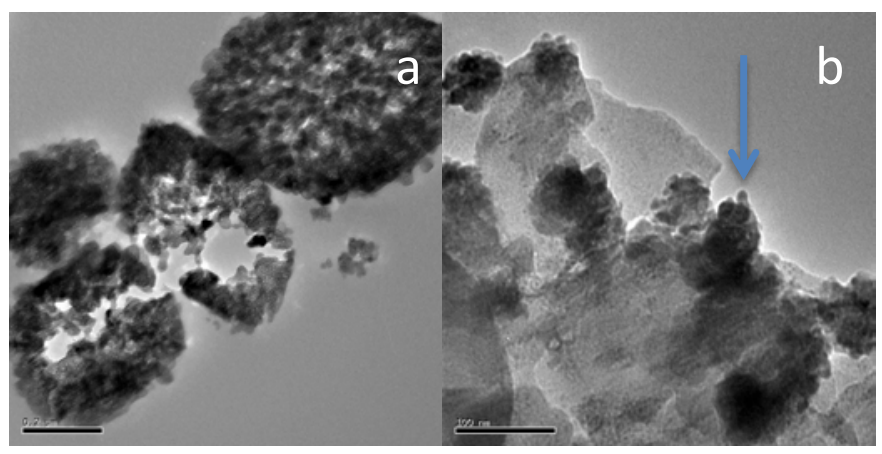

Figure 9. TEM images of barium sulfate formed in the presence of mellitic acid after a) $24 \mathrm{hrs}$ and b) 5 hours (blue arrow highlights one of many smaller particles, which appear to make up the $50 \mathrm{~nm}$ particles)

Figure 10 shows that the nanoparticles do not change size or shape appreciably over time, but rather simply aggregate, suggesting that this is not a dissolution-recrystallization process but a solid-state transformation.

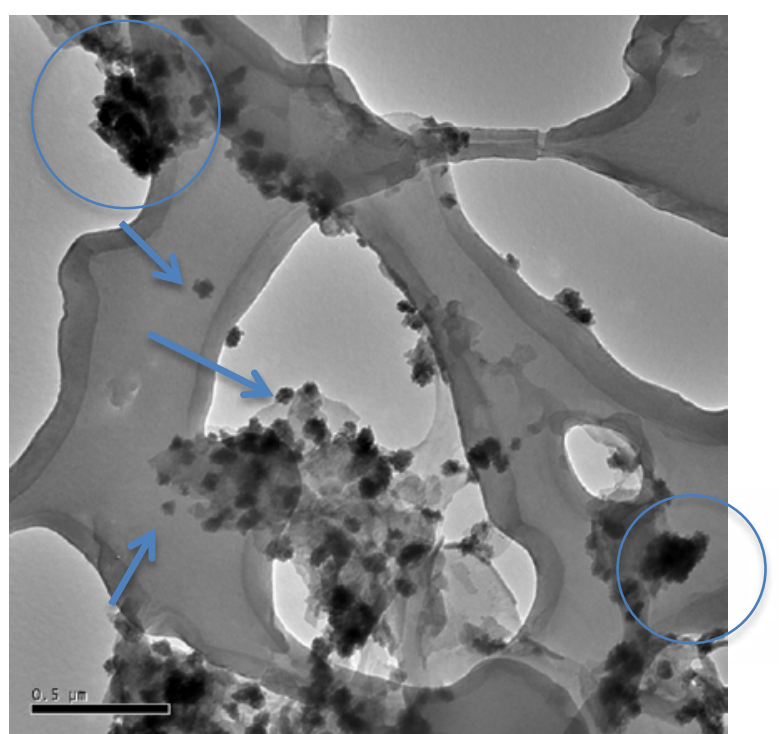

Figure 10. TEM image of solids obtained after 5 hours during crystallization of barium sulfate in the presence of $0.03 \mathrm{mM}$ mellitic 
acid. Blue arrows highlight $\sim 50 \mathrm{~nm}$ nanoparticles, circles highlight self-assembled aggregates.

After nucleation, the $\sim 50 \mathrm{~nm}$ particles self-assemble into almost rhombohedral structures (the evolution of the shape is discussed later). This is at a time when the zeta potential is found to be at its most negative but is mellitic acid concentration dependant. The fact that the surface is negative implies an excess of sulfate or an excess of mellitate ions on the surface. As mellitic acid concentration alters the zeta potential it is reasonable to suggest that the mellitic acid rather than the sulfate is involved here. The infrared spectrum of the mellitic acid during barium suflate crystallization ${ }^{18}$ is consistent with the $\mathrm{L}^{6-} \operatorname{species}^{26}$ meaning that all the carboxylate groups are de-protonated. Thus adsorption of mellitate ions increases the zeta potential. Clearly, these adsorbed ions are not fully screened by barium ions or the surface charge would be more positive. As the concentration of mellitic acid is increased, further adsorption of mellitate would be expected, however further increases in mellitic acid show a decrease in zeta potential. This can only be reconciled if the mode in which mellitate adsorbs changes, either by attracting more barium ions to it (screening the charge) or by changing the physical form in which it adsorbs that results in more carboxylates being incorporated into the growing surface.

At first it may appear surprising that the mellitic acid adsorbs as the completely deprotonated ion. This can be understood in terms of the speciation of the mellitic acid, which at $\mathrm{pH} 6$ while not completely de-protonated has up to $26 \%$ as the $\mathrm{L}^{6-}$ and $62 \%$ $\mathrm{L}^{5-}$ ligand $^{26}$. Thus, adsorption could alter the solution equilibrium such that more and more $\mathrm{L}^{6-}$ is formed as it is removed by the adsorption process until adsorption is no longer favoured. The presence of even small amounts of $\mathrm{L}^{6-}$ ligand may also explain the $2 \mathrm{D}$ nucleation promotion. If complexation with barium ions is preferred with the fully de-protonated ligand, the presence of this small amount of $\mathrm{L}^{6-}$ may promote the de-watering of the barium ion even if it does not significantly impact on the activity of the barium ions in solution.

What is the mesocrystal shape? From the SEM images, the morphology of the particles formed changes only slightly with the concentration as shown in Figure 11 when mellitic acid is present at $0.075 \mathrm{mM}$. As concentration increases, the particles become more elongated and more intergrown solids are seen. In addition, a second population of very small almost rhombic particles begin to appear. The shape of the mesocrystals now becomes clear; although very rounded, the self-assembly process is to form essentially rhombic particles (compare these small mesocrystals to the rhombic particle in Figure 2d). This shape is, in essence, a flattened version of the thermodynamic shape $^{27,28}$. This interpretation is supported by measuring the angles of the less elongated particles which show an obtuse angle of $\sim 105^{\circ}$ compared to the theoretical obtuse angle for the rhombohedron of $101.7^{\circ}$. The elongated particles appear to be developing (010) faces (the flattened ends) and the obtuse angle here is measured to be $\sim 130^{\circ}$, which may imply the (210) faces are becoming less well defined over time. A schematic of the mesocrystal shape is shown in Figure 12.

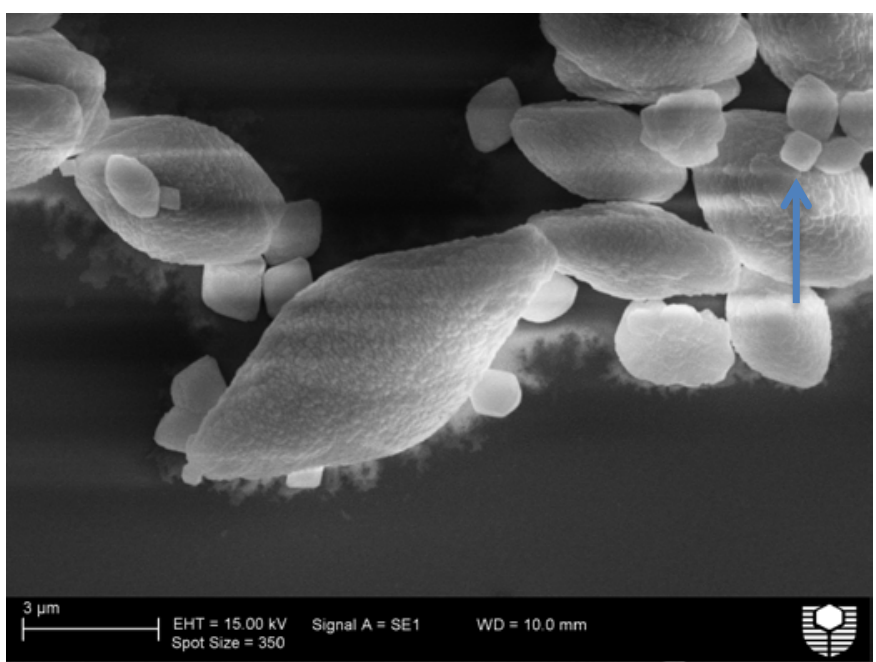

Figure 11. SEM image of barium sulphate mesoparticles formed in the presence of $0.075 \mathrm{mM}$. Blue arrow highlights a rhombic aggregate

Finally, even after mesocrystal formation, the aggregation state also changes at high mellitic acid concentration due to the low zeta potential on the surface of the particles (as can be seen in the supplementary information, S7).

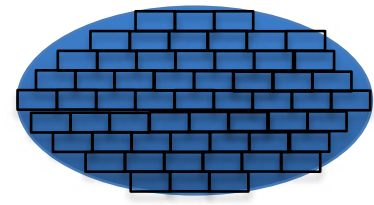

Side view

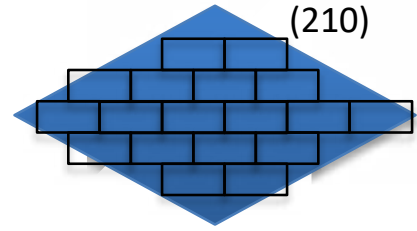

Top view
Figure 12. Schematic of mesocrystal growth showing the 210 diagonal faces in the rhombic particle when viewed from the top

\section{Conclusions}

The mellitic acid can be seen to be an intimate partner during the crystallization of the baryte solids. It appears that it does not inhibit 3D nucleation despite the turbidity results, leading to the formation of small $50 \mathrm{~nm}$ particles, which are themselves aggregates of even smaller particles. At this stage the mellitic acid associated with the solids is the fully de-protonated ligand and results in a minimum surface charge. The presence of mellitic acid also limits the growth of the particles. It can be seen in TEM images that once the particles have approached the $50 \mathrm{~nm}$ size, no further growth occurs and aggregation leads to larger assemblies. These assemblies appear to orient themselves into the thermodynamically stable morphology 
albeit with a short $c$ axis. At high mellitic acid concentration, these assemblies also coagulate due to the low surface charge.

While turbidimetric studies showed a long induction time even with low mellitic acid concentration, implying 3D nucleation inhibition, this may not be the case as the same trend is not observed in DLS measurements. AFM images show many small particles deposited on the surface, too numerous to count showing 2D nucleation promotion and the TEM images support the formation of small nanometric particles of baryte. The promotion of 2D nucleation is most probably due to association of the barium ions with the mellitate ion (which exists in solution at low concentration) resulting in at least partial dewatering of the barium ion.

Of particular interest is that the surface charge measured for the nanoparticles while repulsive is not large, thus surface charge may direct mesocrystal formation but one would assume only for those mellitic acid concentrations between $5 \mathrm{ppm}-10 \mathrm{ppm}$. Above or below these concentrations random coagulation would be expected. It is possible that at short times the surface charge on the $50 \mathrm{~nm}$ particles may be sufficient to induce mesocrystal formation, however, the observation that the mesocrystal morphology is very similar to the thermodynamic morphology may suggest an oriented attachment mechanism unrelated to the surface charge.

\section{Acknowledgements}

The authors acknowledge the use of equipment and technical assistance of the Curtin University Electron Microscope Facility, which has been partially funded by the University, state and Commonwealth Governments, Peter Chapman for providing the thermogravimetric data and Tom Becker for use and technical assistance of the AFM facility at Curtin University.

\section{Notes and references}

a Department of Chemistry, Curtin University, Bentley WA 6102 Australia

${ }^{b}$ Centre for Microscopy, Characterisation and Analysis, The University of Western Australia, Crawley, Western Australia 6009, Australia

\$ Copies of this information may be obtained free of charge from The Director, CCDC, 12 Union Road, Cambridge CB2 1EZ, UK (Fax: + 441223336 033; e-mail: deposit@ccdc.cam.ac.uk or www: http://www.ccdc.cam.ac.uk).

$\dagger$ Electronic Supplementary Information (ESI) available: Complexation as measured by conductivity, barium mellitate crystal structure images, surface roughness shown by line section of height image in AFM, turbidity measured by absorbance at different mellitic acid concentrations, DLS counts measured from timed crystallization experiments and aggregation of particles at high mellitic acid concentration. See DOI: 10.1039/b000000x/

\section{References}

1. Oxtoby, D. W. J. Phys. Condensed Matter, 1992, 4, 7627-7650.

2. Erdimemir, D.; Lee, A. Y.; Myerson, A. S. Accounts of Chemical Research 2009, 42, 621-629.

3. Addadi, L.; Raz, S.; Weiner, S. Advanced Materials, 2003, 15, 959970.

4. Olszta, M. J.; Odom, D. J.; Douglas, E. P.; Gower, L. B. Connective Tissue Research, 2003, 44, 32-334.

5. Niederberger, M.; Cölfen, H. Phys. Chem. Chem. Phys., 2006, 8, 3271-3287.

6. Wang, T.; Reinecke, A.; Cölfen, H. Langmuir, 2006, 22, 8986-8994.

7. Wang, T.; Cölfen, H. Langmuir, 2006, 22, 8975-8985.

8. Yu, S. H.; Antonietti, M.; Cölfen, H.; Hartmann, J. Nano Letters, 2003, 3, 379-382.

9. Qi, L.; Cölfen, H.; Antonietti, M.; Li, M.; Hopwood, J. D.; Ashley, A. J.; Mann, S. Chem. Eur. J., 2001, 7, 3526-3531.

10. Qi, L.; Cölfen, H.; Antonietti, M. Chem. Mater., 2000, 12, 2392 2403.

11. Qi, L.; Cölfen, H.; Antonietti, M. Angew. Chem. Int. Ed., 2000, 39, 604-607.

12. Baynton, A.; Radomirovic, T.; Ogden, M. I.; Raston, C. L.; Richmond, W. R.; Jones, F. CrystEngComm, 2011, 13, 109112.

13. Baynton, A.; Ogden, M. I.; Raston, C. L.; Jones, F. CrystEngComm, 2012, 14, 1057-1062.

14. Ogden, M. I.; Raston, C. L.; Radomirovic, T.; Jones, F. Crystal Growth \& Design, 2014, 14, 1419-1429.

15. Li, M.; Schnablegger, H.; Mann, S. Nature, 1999, 402, 393-395.

16. Ahniyaz, A.; Sakamoto, Y.; Bergstrom, L. PNAS, 2007, 104, 1757017574.

17. Freeman, S. R.; Jones, F.; Ogden, M. I.; Oliviera, A.; Richmond, W. R. Crystal Growth \& Design 2006, 6, 2579-2587.

18. Jones, F. CrystEngComm, 2012, 14, 8374-8381.

19. Baynton, A.; Becker, T.; Chandler, B. D.; Jones, F.; Ogden, M. I.; Radomirovic, T.; Shimizu, G. K. H. CrystEngComm, 2011, 13, 1090-1095.

20. Parkhurst, D. L.; Appelo, C. A., "Users guide to PHREEQC (version 2) - a computer program for speciation, batch reaction, one dimensional transport, and inverse geochemical calculations" U.S Geological Survey Water-Resources Investigation Report 1999, pp 99-4259.

21. Sheldrick, G. M. Acta Crystollographica, 2008, A64, 112-122.

22. Radomirovic, T.; Smith, P.; Jones, F. International Journal of Mineral Processing, 2013, 118, 59-64.

23. Jones, F.; Jones, P.; Ogden, M. I.; Richmond, W. R.; Roh1, A. L.; Saunders, M. Journal of Colloid and Interface Science, 2007, 316, 553-561.

24. Piana, S.; Jones, F.; Gale, J. D. Crystengcomm 2007, 9, 1187-1191.

25. Piana, S.; Jones, F.; Gale, J. D. J. Am. Chem. Soc. 2006, 128, 1356813674.

26. Johnson, B. B.; Sjöberg, S.; Persson, P. Langmuir 2004, 20, 823-828. 
27. Jones, F.; Rohl, A. L. Molecular Simulations, 2005, 31, 393-398.

28. Jones, F.; Richmond, W. R.; Rohl, A. L. J. Phys. Chem. B., 2006, $110,7414-7424$. 